\title{
Perspectives of the study of charmonium and exotics in experiments using antiproton beam with momentum ranging from $1 \mathrm{GeV} / \mathrm{c}$ to $15 \mathrm{GeV} / \mathrm{c}$
}

\author{
M.Yu. Barabanov ${ }^{1}$ \\ Joint Institute for Nuclear Research \\ Joliot-Curie street 6, 141980, Dubna, Moscow region, Russia \\ E-mail: barabanovejinr.ru
}

\section{A.S. Vodopyanov}

Joint Institute for Nuclear Research

Joliot-Curie street 6, 141980, Dubna, Moscow region, Russia

E-mail: vodopianesunhe.jinr.ru

The spectroscopy of charmonium $c \bar{c}$, charmed hybrids $c \bar{c} g$ and tetraquarks $c q \bar{c} \bar{q}^{\prime}, q$ and $\left.\bar{q}^{\prime}=u, d, s\right)$ is discussed. Charmonium and exotics studies are promising for understanding the dynamics of quark interaction at small distances. It is a good testing tool for the theories of strong interactions: QCD in both perturbative and non-perturbative regimes, Lattice QCD, potential models and phenomenological models. The study of strong interactions and hadronic matter in the process of antiproton-proton annihilation seems to be a perspective nowadays. For this purpose the analysis of spectrum of higher lying charmonium, charmed hybrids and tetraquarks is given, and the attempts to interpret a number of experimental data above $D \bar{D}$ threshold are considered. Advantages of the antiproton beam consist in intensive production of particle-antiparticle pairs which is observed in antiproton-proton annihilation. This fact allows one to carry out spectroscopic research with good statistics and high accuracy. The experimental data from different collaborations have been analysed. Especial attention was given to the new states with hidden charm discovered recently. Some of these states may be interpreted as higher laying charmonium states and tetraquarks. But much more data on different decay modes are needed for a deeper analysis. These data can be derived directly from the experiments using the high quality antiproton beam with the momentum ranging from $1 \mathrm{GeV} / \mathrm{c}$ to $15 \mathrm{GeV} / \mathrm{c}$. 
Xth Quark Confinement and the Hadron Spectrum October 8-12, 2012

TUM Campus Garching, Munich, Germany 


\section{Introduction}

The study of strong interactions and hadronic matter in the process of antiproton-proton annihilation seems to be a challenge nowadays. The research of charmonium (the system consisting of charmed quark-antiquark pair $c \bar{c}$ ) and exotic states with the hidden charm such as charmed hybrid (the system consisting of charmed quark-antiquark pair strongly interacting with gluonic component $c \bar{c} g$ ) and tetraquark (the diquark-diantiquark system in which the quarks group into colour-triplet scalar and vector clusters $c q \bar{c} \bar{q}^{\prime}, q$ and $\bar{q}^{\prime}=u, d$, s) spectra, and their main characteristics: mass, width and branching ratios in experiments using antiproton beam with momentum ranging from 1 to $15 \mathrm{GeV} / \mathrm{c}$ at FAIR, is one of the main components of PANDA physical program [1]. The reactions of antiprotons impinging on proton or nuclear targets embedded in the High Energy Storage Ring, will be studied in these experiments.

In the last few years we are witnessing the discovery of a number of new narrow hadronic resonances with charm which do not match standard quark-antiquark interpretation, thereby named exotic hadrons [2]. This has called for alternative interpretations of their inner structure. One of the possible explanations is that these particles are loosely bound molecules of open charm mesons. Another is that new aggregation patterns of quarks in matter are possible. Most of these states were observed above the $D \bar{D}$ threshold in some definite channel (beside $X(3872)$ and $Y(4260)$-states). New particles were produced from $B$-meson decays and in electron-positron or two-photon collisions. Many recently discovered states above the $D \bar{D}$ threshold expect their verification and explanation. Now their interpretation is far from being obvious.

Until recently only few states of charmonium in the mass range from $3 \mathrm{GeV} / \mathrm{c}^{2}$ to $4.5 \mathrm{GeV} / \mathrm{c}^{2}$ have been revealed. The fundamental understanding of strong interactions in terms of QCD was greatly stimulated by the discovery of the vector state of charmonium $J / \Psi$ in 1974 . Till now the charmonium system is considered to be a powerful tool to understand better the nature of strong interactions.Charmonium studies are promising to understand the dynamics of quark interactions at small distances. Charmonium spectroscopy is a good testing tool for the theories of strong interactions: QCD in both perturbative and non-perturbative regimes; QCD inspired purely phenomenological potential models; non-relativistic QCD and LQCD.

In general one can identify four main classes of charmonium decays which are especially interesting and promising from the scientific point of view:

- decays into particle-antiparticle or $D \bar{D}$ pair: $\bar{p} p \rightarrow c \bar{c} \rightarrow \Sigma^{0} \overline{\Sigma^{0}}, \Lambda \bar{\Lambda}$ or $D \bar{D}$ pair;

- decays into light hadrons: $\bar{p} p \rightarrow c \bar{c} \rightarrow \rho \pi, \bar{p} p \rightarrow c \bar{c} \rightarrow \omega \pi^{0}, \bar{p} p \rightarrow c \bar{c} \rightarrow \eta \pi^{0}$;

- decays with $J / \Psi, \Psi^{\prime}$ or $h_{c}$ in the final state: $\bar{p} p \rightarrow c \bar{c} \rightarrow J / \Psi+X \Rightarrow \bar{p} p \rightarrow c \bar{c} \rightarrow J / \Psi \pi^{+} \pi^{-}$, $\bar{p} p \rightarrow c \bar{c} \rightarrow J / \Psi \pi^{0} \pi^{0} ; \quad \bar{p} p \rightarrow c \bar{c} \rightarrow \Psi^{\prime}+X \Rightarrow \bar{p} p \rightarrow c \bar{c} \rightarrow \Psi^{\prime} \pi^{+} \pi^{-}, \quad \bar{p} p \rightarrow c \bar{c} \rightarrow \Psi^{\prime} \pi^{0} \pi^{0} ;$ $\bar{p} p \rightarrow c \bar{c} \rightarrow h_{c}+X=>\bar{p} p \rightarrow c \bar{c} \rightarrow h_{c} \pi^{+} \pi^{-}, \bar{p} p \rightarrow c \bar{c} \rightarrow h_{c} \pi^{0} \pi^{0}$

Charmed hybrids represent themselves as the states with an excited gluonic degree of freedom. Until now, discussions have been focused only around the lowest-lying charmonium hybrids. Four of these states $J^{P C}=2^{-+}, 1^{+}, 1^{--}, 0^{-+}$correspond to a $c \bar{c}$ pair with $J^{P C}=0^{-+}$or $1^{--}$, coupled to a gluon in the lightest mode with $J^{P C}=1^{+-}$. The other four states $J^{P C}=2^{+-}, 1^{+-}, 1^{++}, 0^{+-}$ with the gluon mode $J^{P C}=1^{-+}$are, probably, a bit heavier. Three of these eight charmonium hybrids have spin-exotic quantum numbers $1^{-+}, 0^{+-}, 2^{+-}$, so mixing effects with nearby $c \bar{c}$ states are excluded for them thus making their experimental identification especially easy.

The next possible hybrid states with quantum numbers $2^{++}, 2^{+-}, 1^{++}, 1^{+-}, 0^{+-}, 0^{++}$correspond to $c \bar{c}$ pair with quantum numbers $J^{P C}=1^{+-}$or $J^{P C}=(0,1,2)^{++}$coupled to a gluon in the lightest 
mode with $J^{P C}=1^{+-}$. The states with quantum numbers $2^{--}, 2^{-+}, 1^{--}, 1^{-+}, 0^{-+}, 0^{--}$correspond to $c \bar{c}$ pair with quantum numbers $J^{P C}=1^{+-}$or $J^{P C}=(0,1,2)^{++}$coupled to a gluon mode with $J^{P C}=$ $1^{-+}$. One can find a possibility of the existence of hybrid state with exotic quantum numbers $J^{P C}$ $=0^{--}$.

The most interesting and promising decay channels of charmed hybrids are as follows:

- $\bar{p} p \rightarrow \tilde{\eta}_{c 0,1,2}\left(0^{-+}, 1^{-+}, 2^{-+}\right) \eta \rightarrow \chi_{c 0,1,2}(\eta, \pi \pi ; \ldots)$;

- $\bar{p} p \rightarrow \tilde{h}_{c 0,1,2}\left(0^{+-}, 1^{+-}, 2^{+-}\right) \eta \rightarrow \chi_{c 0,1,2}(\eta, \pi \pi ; \ldots)$;

- $\bar{p} p \rightarrow \tilde{\Psi}\left(1^{-}, 2^{-}\right) \rightarrow J / \Psi(\eta, \omega, \pi \pi, \ldots)$;

- $\bar{p} p \rightarrow \tilde{\eta}_{c 0,1,2}, \tilde{h}_{c 0,1,2}, \tilde{\chi}_{c 1}\left(0^{-+}, 1^{++}, 2^{-+}, 0^{+-}, 1^{+-}, 2^{+-}, 1^{++}, 2^{++}\right) \eta \rightarrow D \bar{D}_{J}^{*} \eta$.

Two generic types of multiquark states have been described in the literature [3]. The first, a molecular state, is comprised of two charmed mesons bound together to form a molecule. These states are by nature loosely bound. Molecular states bind through two mechanisms: quark/colour exchange interactions at short distances and pion exchange at large distance (although pion exchange is expected to dominate). Molecular states are generally not isospin eigenstates, which gives rise to distinctive decay patterns. Since the mesons inside the molecule are weakly bound, they tend to decay as if they are free.

The second type is a tightly bound four-quark state, so called tetraquark that is predicted to have properties that are distinct from those of a molecular state. In the model of Maiani [3], for example, the tetraquark is described as a diquark-diantiquark structure in which the quarks group into colour-triplet scalar and vector clusters and the interactions are dominated by a simple spin-spin interaction. Here, strong decays are expected to proceed via rearrangement processes followed by dissociation that gives rise, for example, to such decays as:

- $\bar{p} p \rightarrow X \rightarrow J / \Psi \rho \rightarrow J / \Psi \pi \pi ; \quad \bar{p} p \rightarrow X \rightarrow J / \Psi \omega \rightarrow J / \Psi \pi \pi \pi, \bar{p} p \rightarrow X \rightarrow \chi_{c J}(1 P) \pi ;$

- $\bar{p} p \rightarrow X \rightarrow D \bar{D}^{*} \rightarrow D \bar{D} \gamma ; \bar{p} p \rightarrow X \rightarrow D \bar{D}^{*} \rightarrow D \bar{D} \eta$.

A prediction that distinguishes tetraquark states containing a $c \bar{c}$ pair from conventional charmonia is possible existence of multiplets which include members with non-zero charge $c u \bar{c} \bar{d}$, strangeness $c d \bar{c} \bar{s}$, or both $c u \bar{c} \bar{s}$.

\section{Discussion of the results of calculation for charmonium, charmed hybrids, tetraquarks}

For this purpose the elaborated analysis of spectrum of the singlet ${ }^{l} S_{0},{ }^{1} P_{l},{ }^{l} D_{2}$ and triplet ${ }^{3} S_{l}$, ${ }^{3} P_{J},{ }^{3} D_{J}$ charmonium states, charmed hybrids with exotic and non-exotic quantum numbers and tetraquarks with the hidden charm in the mass region mainly above $D \bar{D}$ threshold was fulfilled. Different decay modes of charmonium such as decays into particle-antiparticle, baryonantibaryon or $D \bar{D}$ pair, decays into light hadrons and decays with $J / \Psi, \Psi^{\prime}$ and $h_{c}$ in the final state were elaborately investigated. Concerning the charmed hybrids and tetraquarks, the decays into charmonium and light mesons in the final state and decays into $D \bar{D}_{J}^{*}$ pair and $D \bar{D}^{*}$ pair, were, in particular, analyzed. Especial attention is given to the new states with the hidden charm discovered recently (XYZ-particles). The experimental data from different collaborations like Belle, BaBar, BES, CLEO, CDF, D0 were carefully analyzed. Using the combined approach based on the quarkonium potential model and confinement model on a three-dimensional sphere embedded into the four-dimensional Euclidian space [4, 5], twenty two new states of charmonium were predicted in the mass region above $D \bar{D}$-threshold $[6,7]$. More than twenty charmed hybrids [8] and more than twenty tetraquarks are expected to exist in the discussed mass region. The states with the same quantum numbers are possible to consider as radial excited states. The results of calculations for hybrids are in good agreement with the well accepted picture that the quartet $1^{--},(0,1,2)^{-+}$is lower in mass than $1^{++},(0,1,2)^{+-}$. The mass 
splitting between the states $1^{-+}$and $0^{+-}$is about $200 \mathrm{MeV} / \mathrm{c}^{2}$. It has been found that this approach not only predicts new states, but describes the existing experimental data with high accuracy. It was also found that eleven of XYZ-particles could be interpreted as charmonium states and five of them as tetraquarks. The values of the calculated masses coincide (within the experimental error) with the experimentally established values for the $X Y Z$ particles. It was shown that charged/neutral tetraquarks must have their neutral/charged partners with mass values differ by few $\mathrm{MeV}$ (see Fig.1). This assumption can shed light on the nature of neutral $X(3872), X(4350)$ and charged $Z(4050)^{ \pm}, Z(4250)^{ \pm}, Z(4430)^{ \pm}$states. But much more data on different decay modes are needed for deeper analysis. These data can be derived directly from PANDA experiment with its high quality antiproton beam. Hence, there is a possibility of measuring the masses, widths and branching ratios of different charmonium, charmed hybrid and tetraquark states with high accuracy.

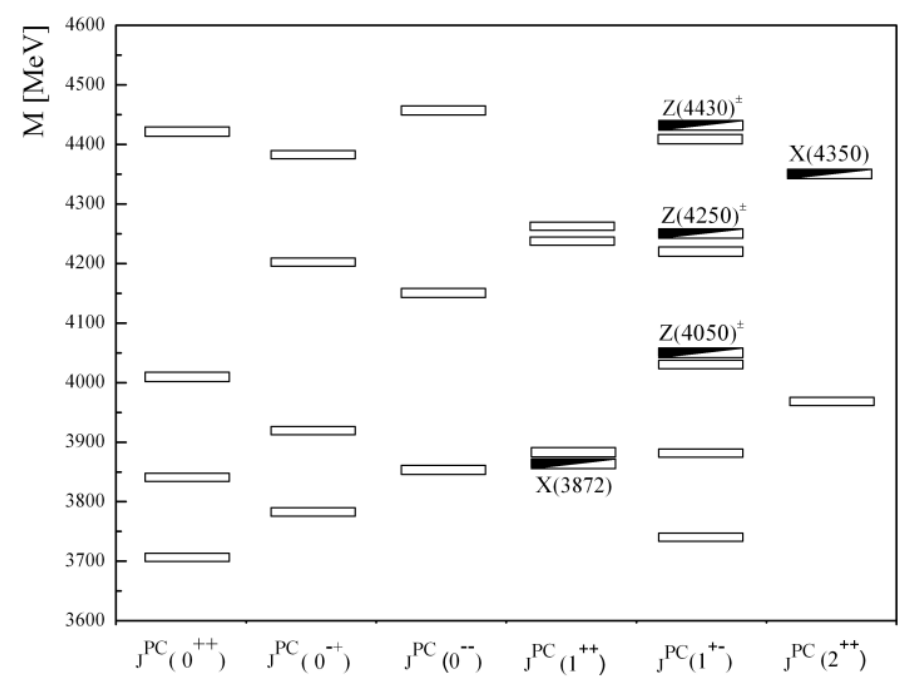

Fig. 1. The spectrum of tetraquarks with hidden charm above $D \bar{D}$ threshold.

The application of the integral approach to calculate the widths of the charmonium, charmed hybrids and tetraquarks with the hidden charm gives results that are in good agreement with the existing experimental data [5,7]. The values of the calculated widths coincide (within the experimental error) with the experimentally established values for the $X Y Z$ particles; the correspondence of the mass values has been discussed above. This fact additionally strongly suggests that some of the $X Y Z$ particles may be interpreted as higher-lying charmonium states and tetraquarks as can be verified by the PANDA experiment.

The branching ratios in the considered decay channels of charmonium, charmed hybrids and tetraquarks were also calculated. Their values are of order $\beta \approx 10^{-1}-10^{-2}$ dependent of their decay channel (mode). From this one can conclude that the branching ratios are significant and searches for charmonium and exotics and studies of the main characteristics of their spectrum seem to be promising for the PANDA experiment at FAIR.

\section{Conclusion}

- A combined approach has been proposed to study the charmonium and exotics on the basis of the quarkonium potential model and confinement model on a three-dimensional sphere embedded into the four-dimensional Euclidian space.

- The most interesting and promising decay channels of charmonium like decays into light hadrons $\bar{p} p \rightarrow c \bar{c} \rightarrow\left(\rho \pi, \omega \pi^{0}, \eta \pi^{0}, \pi \pi\right)$, decays into particle-antiparticle $\bar{p} p \rightarrow c \bar{c} \rightarrow\left(\Sigma^{0} \overline{\Sigma^{0}}\right.$, 
$\Lambda \bar{\Lambda}$ ), decays into $D \bar{D}$-pair, decays with $J / \Psi, \Psi^{\prime}$ and $h_{c}$ in the final state $\bar{p} p \rightarrow c \bar{c} \rightarrow J / \Psi+X$, $\bar{p} p \rightarrow c \bar{c} \rightarrow \Psi^{\prime}+X, \bar{p} p \rightarrow c \bar{c} \rightarrow h_{c}+X$, have been analyzed. Twenty two higher-lying states of charmonium above the $D \bar{D}$ threshold were predicted in the framework of this model.

- Several promising decay channels of the charmed hybrids like decays into charmonium and light mesons in the final state $\bar{p} p \rightarrow c \bar{c} g \rightarrow \chi_{c 0,1,2}(\eta, \pi \pi ; \ldots), \bar{p} p \rightarrow c \bar{c} g \rightarrow J / \Psi(\eta, \omega, \pi \pi ; \ldots)$ and decays into $D \bar{D}_{J}^{*}$ pair $\bar{p} p \rightarrow c \bar{c} g \rightarrow D \bar{D}_{J}^{*} \eta$ were, in particular, considered. More than twenty charmed hybrids with exotic and nonexotic quantum numbers above $D \bar{D}$ threshold are expected to exist in the framework of the combined approach;

- The most interesting and promising decay channels of tetraquarks like decays into charmonium and light mesons in the final state $\bar{p} p \rightarrow X \rightarrow J / \Psi \rho \rightarrow J / \Psi \pi \pi ; \quad \bar{p} p \rightarrow X \rightarrow J / \Psi \omega$ $\rightarrow J / \Psi \pi \pi \pi, \quad \bar{p} p \rightarrow X \rightarrow \Psi(2 S) \pi, \quad \bar{p} p \rightarrow X \rightarrow \chi_{c J}(1 P) \pi$ and decays into $D \bar{D}^{*}$ pair have been analyzed. More than twenty tetraquarks with the hidden charm in the mass region above $D \bar{D}$ threshold are expected to exist in the framework of the combined approach.

- The recently discovered states with the hidden charm above $D \bar{D}$ threshold (XYZ-particles) have been analyzed. Eleven of these states can be interpreted as charmonium (two singlet ${ }^{l} S_{0}$, two singlet ${ }^{1} D_{2}$, three triplet ${ }^{3} S_{l}$, three triplet ${ }^{3} P_{J}$ and one triplet ${ }^{3} D_{J}$ ) and five as tetraquarks (two neutral and three charged). It was shown that charged (neutral) states must have their neutral (charged) partners with mass values differ by few MeV. The necessity of further studying the $X Y Z$-particles and their main characteristics in PANDA experiment with its high quality antiproton beam has been demonstrated.

\section{References}

[1] The PANDA Collaboration: W. Erni et al., Physics Performance Report for PANDA: Strong Interaction Studies with Antiprotons, e-Print: arXiv : 0903.3905 , March, (2009) 63

[2] N. Brambilla et al., Heavy quarkonium: progress, puzzles, and opportunities, Europen Physical Journal, C 71: 1534 (2011) 1

[3] S. Godfrey, S. Olsen, The Exotic XYZ Charmonium-like Mesons, arXiV:0801.3867v1 [hep-ex] (2008)

[4] M.Yu. Barabanov et al., A search for radially excited charmonium states in experiments with low energy antiproton beams, Rusian Physics Journal, V.50, (2007) 1243

[5] M.Yu. Barabanov et al., Application of low energy antiproton beam for charmonium studies, Hadronic Journal, V.32, N.2, (2009) 159

[6] M.Yu. Barabanov, A.S. Vodopyanov, Search for higher lying charmonium states and charmed hybrids in experiments using antiproton beam with momentum ranging from 1 to $15 \mathrm{GeV} / \mathrm{c}$, Physics of Particles and Nuclei Letters, V.8, N.10, (2011) 1069

[7] M.Yu. Barabanov, A.S. Vodopyanov, Study of charmonium and charmed hybrids spectra above $D \bar{D}$ threshold using antiproton beam with momentum from 1 to $15 \mathrm{GeV} / \mathrm{c}$, the Proceedings of the 50 $0^{\text {th }}$ International Winter Meeting on Nuclear Physics, Bormio, Italy, Jan 23-27 (2012) PoS(Bormio2012)008

[8] M.Yu. Barabanov, A.S. Vodopyanov, Application of high quality antiproton beam to study charmonium and exotics above DD threshold, the Proceedings of the XXIV International Conference on Particle Physics and Cosmology "24 Rencontres de Blois", Blois, France, May 27 May-Jun 01 (2012). 